H I G H L I G H T S

\section{CELL MIGRATION}

\section{Stretch and squeeze}

The extension and contraction of muscles that enables us to run (or amble) for the bus has its equivalent in moving cells, which extend and retract sheet-like protrusions known as lamellipodia. As they report in Cell, Michael Sheetz and colleagues have found that, under some circumstances, the lamellipodia stretch out and contract — but do not fully retract - in remarkably regular, repeated cycles, which depend on the length of the lamellipodium. The authors also uncover some of the molecular basis for this rhythmic stretching and squeezing.

Migrating cells use lamellipodia to sense the chemical nature and rigidity of their matrix substrate, treating these local cues as signposts to direct cellular movement. The lamellipodia are 'pushed out' by subcellular networks of actin filaments.

Sheetz and co-workers wanted to find out how matrix rigidity guides lamellipodial protrusion and cell migration. To do so, they started by using total internal reflection fluorescence

\section{CELL CYCLE}

\section{Sisters stick together}

Cells face a sticky problem during the first meiotic division: that of keeping matching sister chromatids glued together while separating them from their homologous counterparts. Watanabe and colleagues, reporting in Nature, and Amon and coworkers, writing in Science, have discovered how the glue between sisters might be preserved.

As cells prepare to undergo meiosis, they duplicate their chromosomes, producing homologous pairs of sister chromatids. The sister chromatids of each pair are glued together, both along their arms and at their centromeres, by a complex that contains the Rec8 protein. During the early stages of meiosis I, the homologues recombine, such that a sister chromatid from one pair becomes attached (through chiasmata) to a sister chromatid from the homologous pair. Then, during anaphase I, the Rec 8 along the chromosome arms must be degraded, so that the chiasmata can be resolved and the homologous pairs can be dispatched to opposite poles of the cell. But the Rec8 at centromeres is maintained, sticking sister chromatids together until meiosis II.

Rec8 is usually expressed only during

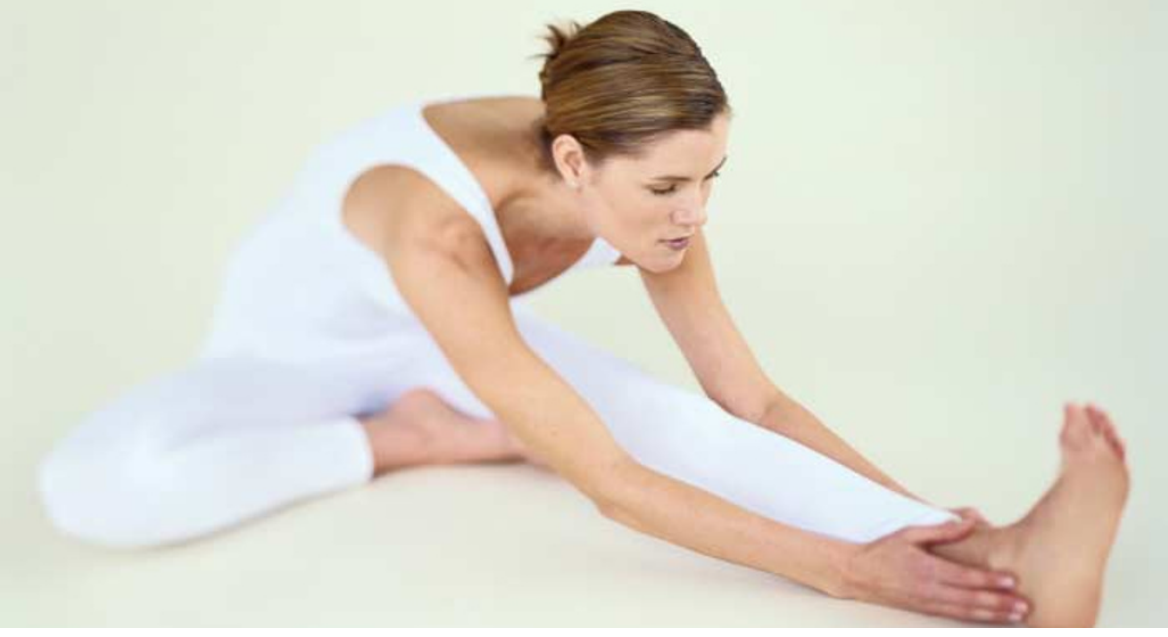

microscopy to look at the contact region between a dye-loaded mouse embryonic fibroblast cell and a glass substrate that was coated with the tough matrix component fibronectin.

Intriguingly, they found that in cells that extend lamellipodia without filopodia (threadlike protrusions that are also often associated with movement), the contact region shrank and grew in a periodic way, which indicates that lamellipodia went through repeated cycles of stretching and squeezing — each cycle lasting about 24 seconds. The net protrusion in each cycle was about $840 \mathrm{~nm}$. By testing the cells on a variety of substrates, Sheetz and colleagues discovered that this periodic behaviour required a

meiosis, but it can also be experimentally expressed during mitosis, when again it glues sister chromatids together. Here, however, it is destroyed along the arms and centromeres simultaneously. So the behaviour of Rec8 during meiosis is clearly not determined only by something intrinsic to the protein.

Instead, Watanabe and co-workers postulated that another protein that is also expressed specifically during meiosis must protect Rec 8 - and that co-expressing this protein with Rec 8 in mitotic cells would inappropriately (and probably fatally) preserve the glue between sister chromatids. So they carried out a screen for such genes in fission yeast. This led them to a previously unknown protein, which they named Sgol (from shugoshin, Japanese for 'guardian spirit').

The authors further found that Sgol is usually expressed only during meiosis in fission yeast, and that it localizes around centromeres. Moreover, when Sgol is deleted, Rec8 labelled with green fluorescent protein (GFP) localizes to centromeres normally, but largely disappears by anaphase I. Sister chromatids then fail to segregate correctly during meiosis II - probably because the premature loss of centromeric cohesion disrupts proper attachment to the chromosome-segregating machinery.

By searching genome databases for relatives of Sgol in other organisms, Watanabe and colleagues found another fission yeast protein, rigid matrix: very soft surfaces would not do.

Using a laser trap and a bead attached to the edge of a lamellopodium, the authors deduced that the interruptions in lamellipodial extension were driven by an increase in the rate of retraction of the underlying actin network (this network is continually retracting in lamellipodia, but during extension the rate of polymerization at the front exceeds the retraction rate). Sheetz and co-workers also investigated the role of integrins - transmembrane receptors for matrix components - by studying the localization of integrin- $\beta 3$ attached to green fluorescent protein. They found that this receptor formed rows in a periodic manner, every 23 seconds or so.

Sgo2, which seems to be required during mitosis; one homologue in budding yeast; and some more distant relatives in fruitflies, nematodes, plants, mice and humans. A screen for mutant budding yeast strains that show aberrant meiotic chromosome segregation also led Amon and colleagues to Sgol, and to two further proteins, $\operatorname{Iml} 3$ and Chl4. These authors show that Rec 8 is also prematurely lost from centromeres in Sgo1deficient meiotic budding yeast, and (to a lesser extent) in cells lacking $\mathrm{Iml} 3$ or $\mathrm{Chl} 4$. And they find that budding yeast Sgol usually localizes to centromeres. The implication is that Sgol indeed protects centromeric Rec8 from degradation. Curiously, the counterpart of Sgol in fruitflies, Mei-S332, was already suspected to be involved in protecting centromeric cohesion, but had been discounted because of a lack of known counterparts in other species.

It remains to be seen exactly how Sgo 1 works. Does it act as a physical shield, standing between Rec8 and the degradation enzyme? Or does it inhibit this enzyme? There are plenty of questions left to tackle.

Amanda Tromans

30) References and links

ORIGINAL RESEARCH PAPERS Kitajima, T. S ,

Kawashima, S. A. \& Watanabe, Y. The conserved kinetochore protein shugoshin protects centromeric cohesion during meiosis. Nature 427, 510-517 (2004)| Marston, A. L.,

Tham, W.-H., Shah, H. \& Amon, A. A genome-wide screen identifies genes required for centromeric cohesion. Science 29 January 2004 (doi:10.1126/science.1094220) 
So what triggers the increased rate of retraction that underlies contraction? Sheetz and colleagues suggest that a molecule hitches a ride on the retreating actin filaments, from the front of the lamellipodium to the rear, signalling contraction when it gets to the back. The authors find that $\alpha$-actinin and myosin-light-chain kinase (MLCK) are transported in this way, reaching the back of the lamellipodium in around 25 seconds in normal lamellipodia. They propose that MLCK could be a contraction-triggering signal, as inhibiting this enzyme significantly reduced the duration of, or even eliminated, the extension-contraction phase.

Now we need to know why cells behave in this way on tough substrates - is it, as Sheetz and coworkers suggest, that regular periods of contraction enable the locally protruding cell edge to get a better grip on the surface, allowing greater extension towards rigid regions? Is MLCK indeed a signal that triggers squeezing? And could the directed movement of signals along cytoskeletal filaments occur in other contexts, too?

\section{(9) References and links}

ORIGINAL RESEARCH PAPER Giannone, G. et al. Periodic

lamellipodial contractions correlate with rearward actin waves. Cell 116, 431-443 (2004)
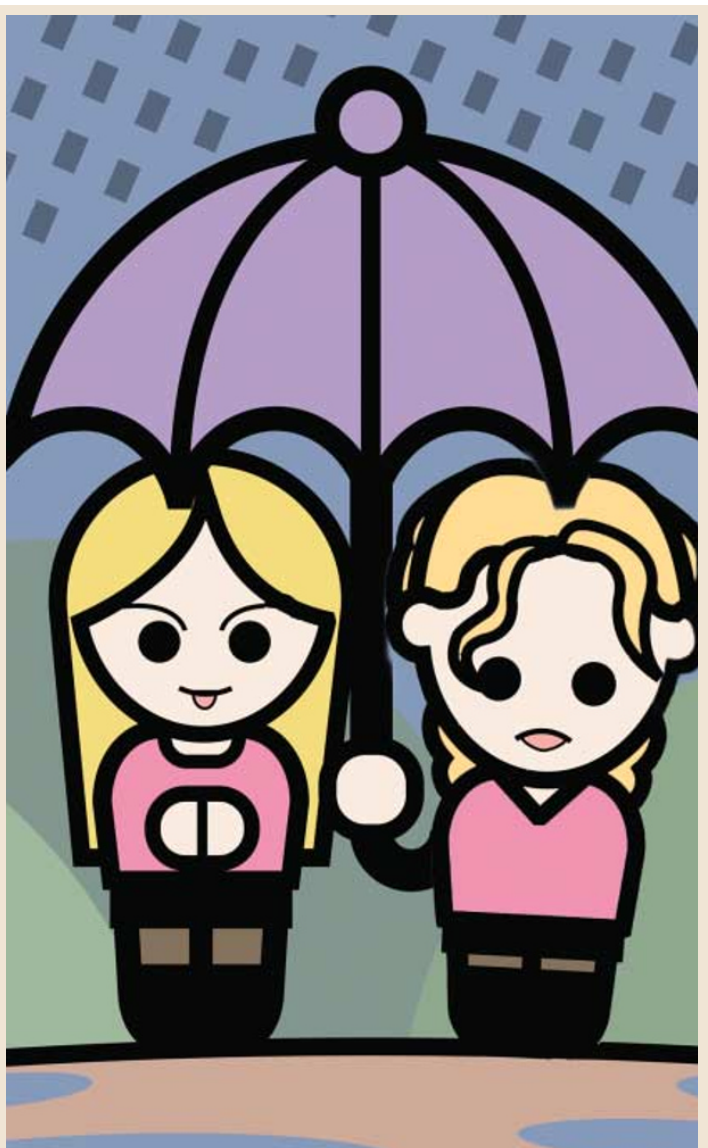

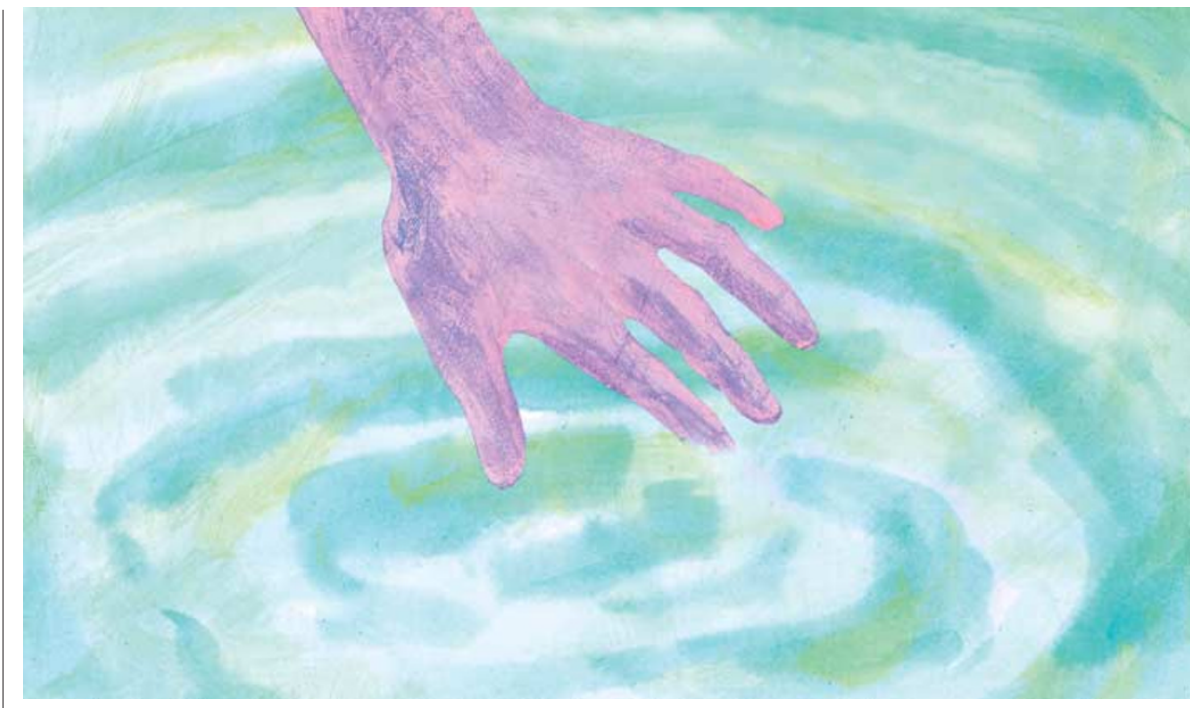

\section{CELL GROWTH}

\section{Testing the water}

If you were looking for a mate, how would you find one? The budding yeast Saccharomyces cerevisiae has a simple solution. It forms mating projections - polarized cell-surface structures that are involved in cell-cell recognition - that allow it to search for a partner. These projections normally grow towards cells of the opposite sex by responding to pheromone gradients, but when pheromone levels are high, periodic growth occurs in random directions. Bidlingmaier and Snyder now suggest that this allows yeast to 'test the water' to find nearby mating partners in the absence of a pheromone gradient. And, they show that the actin-polymerizing polarisome complex and the Rhorelated GTPase $\mathrm{Cdc} 42$ regulate this process.

Yeast cells were treated with high concentrations of the $\alpha$-factor pheromone, which induces periodic mating projections, and were observed by time-lapse photography. When a new projection appeared, the existing projections ceased to grow, which indicates that these two processes are closely linked. So, as the actin cytoskeleton is involved in polarized growth, the authors blocked actin polymerization - and hence the growth of the mating projections - by briefly treating the cells with an actin-polymerization inhibitor. Transient disruption of the actin cytoskeleton produced new projections in the treated cells, which confirmed that termination and initiation of projection growth are tightly linked. But what controls this process?

The polarisome complex, which comprises Spa2, Pea2, Bniland Bud6, is important for polarized growth. Spa2 has been implicated in the periodic initiation of polarized growth, so the authors investigated whether the proteins of the polarisome complex regulate mating projection. Projections were formed less frequently and were wider, and faster growing, in spa $2 \Delta$, pea $2 \Delta$ and bnil 1 , but not bud6 $6 \Delta$, mutants compared with wild-type cells, which indicates that Spa2, Pea2 and Bnil are involved in controlling the timing and frequency of mating-projection formation. The authors were surprised to find that growth termination was delayed in the spa $2 \Delta$, pea $2 \Delta$ and bni $1 \Delta$ mutants, which indicates that these proteins are also required to terminate growth.

As Bnil is a downstream effector of the Cdc42 GTPase, the authors next investigated components of the Cdc42 signalling pathway. They found that $\mathrm{Cdc} 42$ and its regulators $\mathrm{Cdc} 24$ and Bem 3 also control the frequency of projection formation. Interestingly, Bem 3 did not affect growth termination, which indicates that initiation and termination might be regulated by distinct, but overlapping pathways. The lack of Fus 1 - a protein required for mating inhibited growth termination, but not growth initiation, providing further evidence that the pathways are partially separated.

The authors propose that Cdc42 phosphorylation activates Bnil in the polarisome complex, which then promotes projection initiation and, at the same time, terminates the growth of existing projections. As Bem 3 and Fus1 only affect part of this process, at least one other regulatory pathway must exist. Many biological processes involve cell polarization, so it will be interesting to see if the proposed mechanism is a general one.

\section{(2) References and links}

Emma Croager ORIGINAL RESEARCH PAPER Bidlingmaier, S. \& Snyder, M. Regulation of polarized growth initiation and termination cycles by the polarisome and Cdc42 regulators. J. Cell Biol. 164 207-218 (2004) WEB SITE

Michael Snyder's laboratory: http://www.yale.edu/snyder/ 